SubmitTed V2 April 30, 2014

Preprint typeset using LTEX style emulateapj v. 5/2/11

\title{
NEW PERSPECTIVE ON GALAXY OUTFLOWS FROM THE FIRST DETECTION OF BOTH INTRINSIC AND TRAVERSE METAL-LINE ABSORPTION
}

\author{
Glenn G. Kacprzak ${ }^{1,2}$, Crystal L. Martin ${ }^{3}$, Nicolas Bouché ${ }^{4,5}$, Christopher W. ChurChill ${ }^{6}$, JefF Cooke $^{1}$, \\ Audrey LeReun ${ }^{4,5}$, Ilane Schroetter ${ }^{4,5}$, Stephanie H. Ho $^{3}$, Elizabeth KlimeK ${ }^{6}$ \\ Submitted V2 April 30, 2014
}

\begin{abstract}
We present the first observation of a galaxy $(z=0.2)$ that exhibits metal-line absorption back-illuminated by the galaxy ("down-the-barrel") and transversely by a background quasar at a projected distance of $58 \mathrm{kpc}$. Both absorption systems, traced by MgII, are blueshifted relative to the galaxy systemic velocity. The quasar sight-line, which resides almost directly along the projected minor axis of the galaxy, probes $\mathrm{Mg}$ I and $\mathrm{Mg}$ II absorption obtained from Keck/LRIS and Ly $\alpha$, Si II and Si III absorption obtained from HST/COS. For the first time, we combine two independent models used to quantify the outflow properties for down-the-barrel and transverse absorption. We find that the modeled down-the-barrel deprojected outflow velocities range between $V_{d t b}=45-255 \mathrm{~km} \mathrm{~s}^{-1}$. The transverse bi-conical outflow model, assuming constant-velocity flows perpendicular to the disk, requires wind velocities $V_{\text {out flow }}=40-80 \mathrm{~km} \mathrm{~s}^{-1}$ to reproduce the transverse Mg II absorption kinematics, which is consistent with the range of $V_{d t b}$. The galaxy has a metallicity, derived from $\mathrm{H} \alpha$ and $\mathrm{N}$ II, of $[\mathrm{O} / \mathrm{H}]=-0.21 \pm 0.08$, whereas the transverse absorption has $[\mathrm{X} / \mathrm{H}]=-1.12 \pm 0.02$. The galaxy star-formation rate is constrained between $4.6-15 \mathrm{M}_{\odot} \mathrm{yr}^{-1}$ while the estimated outflow rate ranges between 1.6-4.2 $\mathrm{M}_{\odot} \mathrm{yr}^{-1}$ and yields a wind loading factor ranging between $0.1-0.9$. The galaxy and gas metallicities, the galaxy-quasar sight-line geometry, and the down-the-barrel and transverse modeled outflow velocities collectively suggest that the transverse gas originates from ongoing outflowing material from the galaxy. The $\sim 1$ dex decrease in metallicity from the base of the outflow to the outer halo suggests metal dilution of the gas by the time it reached $58 \mathrm{kpc}$.
\end{abstract}

Subject headings: galaxies: halos — galaxies: intergalactic medium — quasars: absorption lines

\section{INTRODUCTION}

Galactic-scale outflows appear to be quite common amongst star-forming galaxies and these outflows are thought to contribute significantly to the metal enrichment of the universe (e.g., Oppenheimer et al. 2010). Cool gas tracers such as the Mg II $\lambda \bar{\lambda} 2796,2803$ doublet are commonly used to trace and measure outflow properties.

Star-forming galaxies show intrinsic blueshifted outflowing Mg II gas with velocities of $100-1000 \mathrm{~km} \mathrm{~s}^{-1}$ (e.g., Tremonti et al. 2007; Weiner et al. 2009; Martin \& Bouché 2009; Coil et al. 2011; Martin et al. 2012; Rubin et al. 2013) and occasionally show redshifted infalling gas with velocities of 50-200 km s ${ }^{-1}$ (e.g., Rubin et al. 2012; Martin et al. 2012; Rubin et al. 2013). These studies of systems back-illuminated by the galaxy ("down-the-barrel") have shown that outflowing gas velocities weakly correlate with galaxy star-formation rates (SFR) and specific star-formation rates (Martin 2005; Weiner et al. 2009; Martin et al. 2012; Rubin et al. 2013). The outflow velocities also correlate with galaxy inclination, with higher velocities occurring for face-on galaxies (Kornei et al. 2012; Bordoloi et al. 2013). Although these observations constrain wind velocities and how they relate to

\footnotetext{
${ }^{1}$ Swinburne University of Technology, Victoria 3122, Australia gkacprzak@astro.swin. edu. au

${ }^{2}$ Australian Research Council Super Science Fellow

${ }^{3}$ Physics Department, University of California, Santa Barbara, CA 93106, USA

${ }^{4}$ CNRS, Institut de Recherche en Astrophysique et Planétologie [IRAP] de Toulouse, 14 Avenue E. Belin, F-31400 Toulouse, France

${ }^{5}$ Université Paul Sabatier de Toulouse, UPS-OMP, IRAP, F-31400 Toulouse, France

${ }^{6}$ New Mexico State University, Las Cruces, NM 88003
}

their galaxies, they do not constrain their extent or the mass ejection rates.

Background quasars have been used to probe the "transverse" absorption associated with outflows, which is supported by a strong azimuthal dependence of $\mathrm{Mg}$ II absorption around galaxies (Bouché et al. 2012; Kacprzak et al. 2012; Bordoloi et al. 2014). Bouché et al. (2012) have also shown that transverse absorption detected along the projected minor axes of galaxies is kinematically consistent with simple biconical outflows, leading to constraints on mass outflow rates. The azimuthal dependence, together with an inclinationdependence in down-the-barrel studies, points towards a picture where outflows are well collimated with half-opening angles of $\sim 45$ degrees (Bouché et al. 2012; Kacprzak et al. 2012; Martin et al. 2012; Bordoloi et al. 2013; Bordoloi et al. 2014).

Simulations suggest that the metallicity of transverse absorption can also be used to constrain its origins (e.g., Shen et al. 2012). Low metallicity gas near galaxies has been assumed to be infalling (e.g., Kacprzak et al. 2012; Bouché et al. 2013), while high metallicity gas near galaxies has also been assumed to be outflowing (Péroux et al. 2011; Stocke et al. 2013).

Despite the detailed and large studies of transverse and down-the-barrel absorption systems, the two have yet to be directly connected. We have begun a Keck-SDSS QSOgalaxy pair survey, expanding on the pioneering work of Barton \& Cooke (2009), to study the CGM using Mg II absorption around $z=0.1-0.3$ galaxies. Here we present our initial findings of the first observation that directly connects down-the-barrel outflows with the transverse gas observed $58 \mathrm{kpc}$ from a $z=0.2$ galaxy. In $\S 2$ we describe the data 

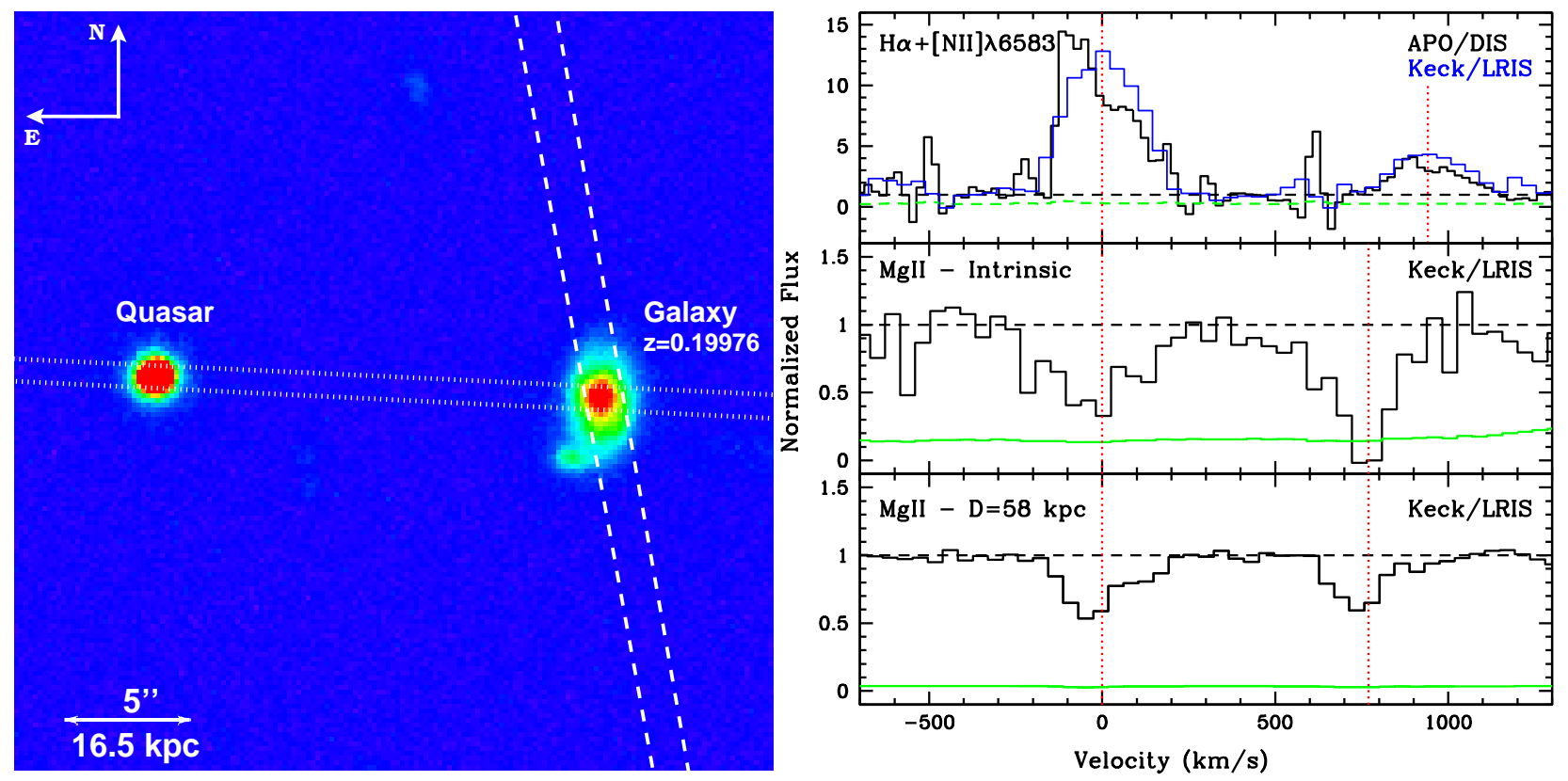

FIG. 1.- (left) $30^{\prime \prime} \times 30^{\prime \prime} i$-band MegaCam/CFHT image of the background quasar and the $\mathrm{z}=0.19976 \mathrm{Mg}$ II absorbing galaxy at a projected distance of $58 \mathrm{kpc}$. The dashed-line shows the DIS/APO slit placed along the galaxy major-axis and the dotted-line shows the LRIS/Keck slit placed along the minor-axis. - (right, top) $\mathrm{H} \alpha+[\mathrm{N}$ II] emission along the major axis (DIS/APO, black) and along the minor axis (Keck/LRIS, blue). In all panels, red vertical-lines define the $\mathrm{H} \alpha$ centroid and galaxy systemic velocity. (right, middle) LRIS/Keck spectrum of the Mg II $\lambda \lambda 2796,2803$ absorption "down-the-barrel" of the galaxy, which is blueshifted relative to $\mathrm{H} \alpha$. (right, bottom) $\mathrm{Mg}$ II $\lambda \lambda 2796,2803$ absorption along the quasar sight-line, which is also blueshifted.

and our analysis. In $\S 3$, we present kinematic models of the down-the-barrel and transverse absorption profiles and show that they are both consistent with wind models. We use $H S T / C O S$ observations to compute the metallicity of the transverse gas. We end with a discussion and concluding remarks in $\S 4$. We adopt a $h=0.70, \Omega_{\mathrm{M}}=0.3, \Omega_{\Lambda}=0.7$ cosmology.

\section{DATA AND ANALYSIS}

In Figure 1, we show our targeted foreground galaxy ( $\mathrm{z}=0.19976$ ), producing the observed $\mathrm{Mg}$ II absorption, and background quasar $(\mathrm{z}=0.77) \mathrm{J} 165931+373528$ that are separated by $58 \mathrm{kpc}$ projected, on the sky. Below we describe the data acquired for our analysis.

\subsection{Galaxy and Quasar Spectroscopy}

The galaxy and background quasar spectra were obtained on 2013 April 11 using the Keck Low Resolution Imaging Spectrometer (LRIS; Oke et al. 1995) with the blue 1200 lines $/ \mathrm{mm}$ grism blazed at $3400 \AA$ providing wavelength coverage from the atmospheric cut-off to $3890 \AA$. We further used the red 900 lines $/ \mathrm{mm}$ grating with a central wavelength of $7750 \AA$. We used a $1^{\prime \prime}$ slit and a spectral binning of two, providing a dispersion of $0.48 \AA$ pix $^{-1}$ and $\sim 1.6 \AA$ resolution $\left(\sim 145 \mathrm{~km} \mathrm{~s}^{-1}\right)$ in the blue and a dispersion of $1.06 \AA \mathrm{pix}^{-1}$ and $\sim 3.5 \AA$ resolution $\left(\sim 140 \mathrm{~km} \mathrm{~s}^{-1}\right)$ in the red. The slit was placed such that it spatially covered both the galaxy and the quasar (Figure 11). The total of integration time was 2240s.

An additional galaxy spectrum was obtained on $2013 \mathrm{Au}-$ gust 12 using the Double Imaging Spectrograph (DIS) at the Apache Point Observatory (APO) 3.5-m telescope. We placed the $1.5^{\prime \prime}$ slit along the galaxy major axis (Figure 1) to obtain the kinematic velocity zeropoint from the $\mathrm{H} \alpha$ emissionline. We used the R1200 grating, providing a dispersion of
$0.58 \AA \mathrm{pix}^{-1}$ and $\sim 1.3 \AA$ resolution $\left(\sim 50 \mathrm{~km} \mathrm{~s}^{-1}\right)$. The wavelength coverage is $1160 \AA$ and we used a wavelength centroid of $8100 \AA$. The total of integration time was 4400 s.

We supplement our data with a $H S T / C O S$ quasar spectrum taken with the G160M grating (PI:Nestor, PID:12593) in order to measure the physical properties of the transverse gas derived from Ly $\alpha$, Si II, Si III, Mg II and Mg I absorption. The total exposure time is 2100 s and has a wavelength coverage of $1408-1776 \AA$.

All the spectra were reduced using standard techniques and were heliocentric and vacuum corrected. The spectral analysis was performed using our in-house software. Transverse absorption-line rest-frame equivalent widths are listed in Table 1.

\subsection{Galaxy Imaging}

In Figure 1 we show $30^{\prime \prime} \times 30^{\prime \prime}$ section of a $1981 \mathrm{~s} i$-band image obtained using MegaCam and was produced by the MegaPipe pipeline (Gwyn 2012), which has a spatial resolution of $0.186^{\prime \prime} \mathrm{pix}^{-1}$. We used our own custom MCMC bayesian code to determine galaxy morphological parameters by fitting a Sérsic profile convolved with the image point spread function that produced a Sérsic index of $n=0.91 \pm 0.01$, a disk effective radius of $r_{e}=1.54 \pm 0.01^{\prime \prime}$ and an inclination of $i=52 \pm 5$ degrees. The quasar is nearly aligned with the galaxy projected minor axis with a galaxy position angle of $P A=-3.5 \pm 0.3$ degrees, which provides ideal geometry to probe galactic winds (Bordoloi et al. 2011; Bouché et al. 2012; Kacprzak et al. 2012). The galaxy/quasar projected separation is $D=17.53^{\prime \prime}(57.8 \mathrm{kpc})$.

\subsection{Galaxy Star Formation Rate}

We estimate lower limits on the unobscured star formation rate (i.e., not extinction corrected) using both the $\mathrm{H} \alpha$ flux 


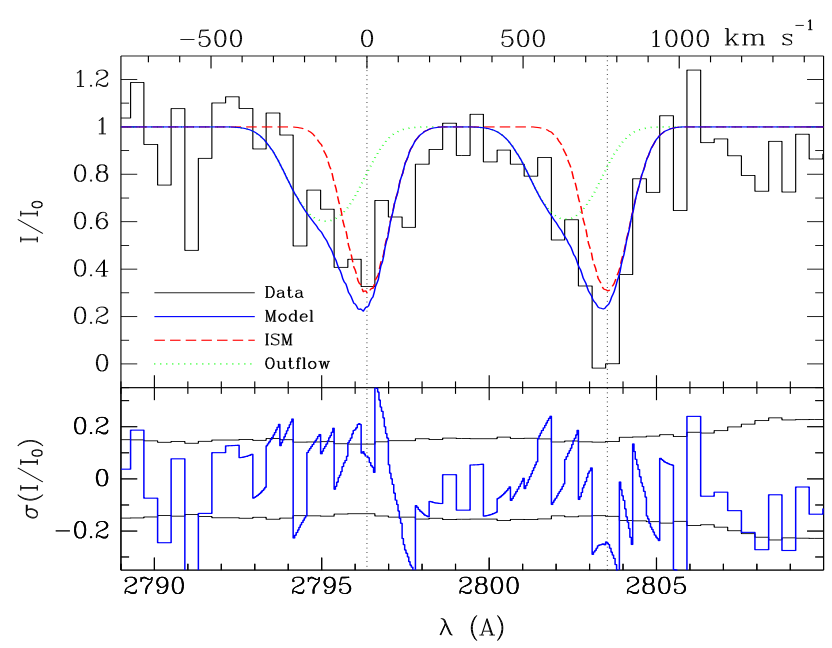

FIG. 2. - The down-the-barrel Mg II $\lambda \lambda 2796,2803$ doublet absorption is fit using two components. One component is fixed to galaxy systemic velocity (vertical dotted-line) having a fixed velocity width of $200 \mathrm{~km} \mathrm{~s}^{-1}$ (representing the ISM - red dashed line) and another component having a variable velocity width representing the outflowing gas (green dotted-line). Below is the fit residuals with one sigma errors. The outflow absorption component centroid is blueshifted by $132 \pm 25 \mathrm{~km} \mathrm{~s}^{-1}$. For gas launched perpendicular to the disk, this corresponds to a wind velocity of $214 \pm 41 \mathrm{~km} \mathrm{~s}^{-1}$.

within the LRIS spectroscopic aperture and the flux density measured from a GALAX NUV image covering rest-frame wavelengths from 1476 to $2359 \AA$. The SFR of $4.6 \mathrm{M}_{\odot} \mathrm{yr}^{-1}$ derived from the GALAX UV continuum measurement following Kennicutt (1998) provides the more accurate lower bound on the SFR because a substantial aperture correction must be applied to obtain the total $\mathrm{H} \alpha$ galaxy flux. Alternatively, fitting the $22 \mu \mathrm{m}$ flux or the UV-to-17 $\mu \mathrm{m}$ SED with the CIGALE code (Noll et al. 2009), we estimate the dustcorrected SFR could be as large as $15 \mathrm{M}_{\odot} \mathrm{yr}^{-1}$ and a stellar mass of $\log M_{*}=10.6 \pm 0.07$.

\section{RESULTS}

In Figure 1, we show the first known case where Mg II absorption is detected at: (1) intrinsic to the galaxy (i.e., "downthe-barrel") and (2) along the quasar sight-line $58 \mathrm{kpc}$ away (i.e., "transverse"). The velocity zeropoint is set by the $\mathrm{H} \alpha$ emission-line centroid, taken along the major axis with DIS, was determined to be $z=0.19976 \pm 0.00003$. This is consistent with the minor axis $\mathrm{H} \alpha$ centroid obtained with LRIS. Note that the down-the-barrel Mg II absorption is blueshifted relative to the galaxy systemic velocity. The down-the-barrel restframe $\mathrm{Mg}$ II equivalent widths are $W_{r}(2796)=0.73 \pm 0.31 \AA$ and $W_{r}(2803)=1.47 \pm 0.26 \AA$. The observed doublet ratio is less than unity, for this likely saturated system, which could be due to optically thin Mg II emission from gas within/around the galaxy (Martin et al. 2012).

The transverse MgII absorption also exhibits a blueshift relative to the galaxy systemic velocity. The transverse restframe Mg II equivalent widths are $W_{r}(2796)=0.88 \pm 0.04 \AA$ and $W_{r}(2803)=0.70 \pm 0.04 \AA$. Given the relative blueshifts, we explore whether outflows originating from the galaxy can explain the kinematics of the transverse absorption at $58 \mathrm{kpc}$.

\section{1. "Down-the-Barrel” Absorption Wind Model}

TABLE 1

TRANSVERSE ABSORPTION PROPERTIES

\begin{tabular}{llcr}
\hline \hline \multicolumn{1}{c}{ Ion } & $\begin{array}{c}\text { Telescope/ } \\
\text { Instrument }\end{array}$ & $\begin{array}{c}W_{r} \\
(\AA)\end{array}$ & $\log [N(\mathrm{X})]$ \\
\hline Ly $\alpha$ & $H S T / C O S$ & $2.03 \pm 0.07$ & \\
H I & & & $18.89 \pm 0.15$ \\
Mg II $\lambda 2796$ & Keck/LRIS & $0.88 \pm 0.04$ & \\
Mg II $\lambda 2803$ & Keck/LRIS & $0.70 \pm 0.04$ & \\
Mg II & & & $13.62 \pm 0.18$ \\
Mg I $\lambda 2853$ & Keck/LRIS & $0.13 \pm 0.04$ & \\
Mg I & & & $12.05 \pm 0.13$ \\
Si II $\lambda 1190$ & $H S T / C O S$ & $0.33 \pm 0.05$ & \\
Si II $\lambda 1193$ & $H S T / C O S$ & $0.37 \pm 0.04$ & \\
Si II $\lambda 1260$ & $H S T / C O S$ & $0.44 \pm 0.06$ & \\
Si II & & & $13.93 \pm 0.07$ \\
Si III $\lambda 1207$ & $H S T / C O S$ & $0.42 \pm 0.05$ & \\
Si III & & & $13.54 \pm 0.06$ \\
SiIV $\lambda 1394$ & $H S T / C O S$ & $<0.62$ & \\
Si IV $\lambda 1403$ & $H S T / C O S$ & $<0.59$ & \\
SiIV & & & $<12.80$ \\
\hline
\end{tabular}

The velocity of the MgII absorption is notably blueshifted relative to the galaxy systemic velocity. A single Gaussian fit to the data shows that the absorption is blueshifted by $43 \pm 15 \mathrm{~km} \mathrm{~s}^{-1}$. In order to estimate the bulk velocity of the Mg II wind component, we applied a two-component absorption model. One component is fixed to systemic velocity of the galaxy with a fixed velocity width of $200 \mathrm{~km} \mathrm{~s}^{-1}$, as measured from galaxy $\mathrm{H} \alpha$ emission-line (representing the ISM), and one component that has a variable velocity width that represents the outflowing gas (Martin et al. 2012). The model was convolved with a Gaussian profile to model the LRIS instrumental spread function (ISF). The covering fraction of the variable velocity component is fixed to unity. The doublet ratio is free parameter and the residuals between the model and the data are minimized iteratively using the LevenbergMarquardt algorithm (Press et al. 1992). In Figure 2 we show the best resulting model fit. As noted, the ISM component of the fit is at the systemic velocity of the galaxy while the outflow component centroid is blueshifted by $132 \pm 25 \mathrm{~km} \mathrm{~s}^{-1}$.

We note that the velocity offset computed above may not directly represent the outflow velocity component since the ratio of equivalent widths is nonphysical, which leads us to suspect the absorption is partially filled in by resonance $\mathrm{Mg}$ II emission. To test this, we apply models containing an additional emission component. Since the data do not resolve an emission component, we attempt to gain insight by assuming that the emission is at the systemic velocity and its amplitude is fixed at a modest, but random, value. Our models show that the addition of the emission component does not significantly improve the fit statistic, although we find that including it tends to lower the inferred outflow velocity.

In summary, our single and double-line models indicate that the line-of-sight outflow velocity component centroid likely ranges between $28-157 \mathrm{~km} \mathrm{~s}^{-1}$.

\subsection{Transverse Absorption Wind Model}

Here we explore whether or not the MgII absorption detected at $58 \mathrm{kpc}$ is consistent with an outflow model. We adopt a bi-conical outflow (Bouché et al. 2012) since the gas trajectories will tend to open up from the internal wind pressure into a cone-like shape (Shen et al. 2012). The model has two free 

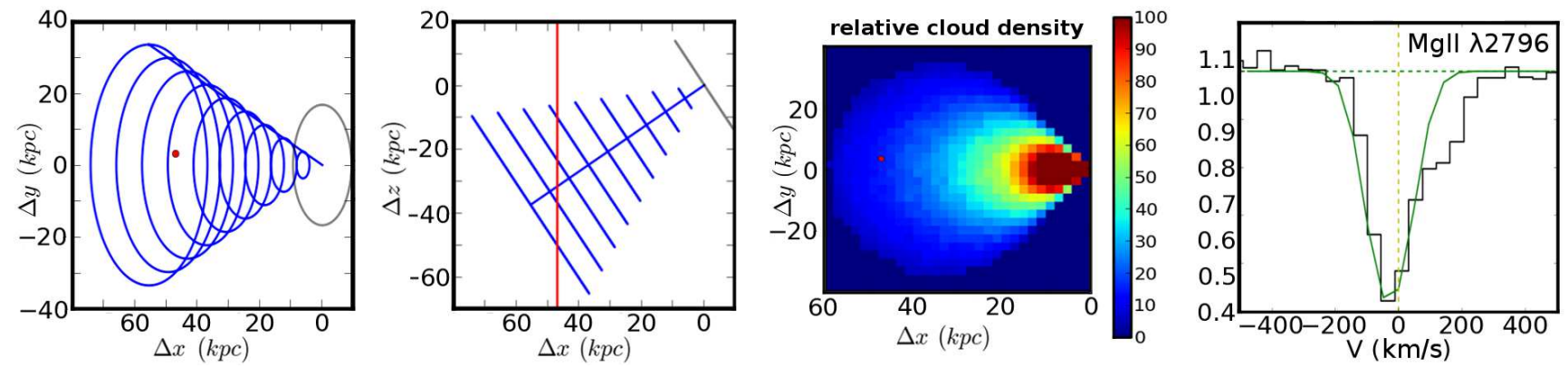

FIG. 3.- (left) Kinematic conical wind model viewed on the sky-plane where the $x$-axis corresponds to the minor axis and the $y$-axis to the major axis. The grey oval represents an inclined galaxy and the circles (blue) represent the conical outflow. The red point represents the background quasar. (middle-left) Kinematic model shown along the quasar sight-line ( $z$-axis) indicated by the solid red line. (middle-right) The average cloud relative density map with the red point indicating the quasar sight-line. (right) The Mg II $\lambda 2796$ transverse absorption (black histogram) with the distribution of the cloud line-of-sight velocities (convolved with the LRIS resolution) at the quasar location (green). The shape, width, and velocity offsets of the synthetic Mg II absorption constrain the model to have cloud outflow velocities of $V_{\text {out flow }}=40-80 \mathrm{~km} \mathrm{~s}^{-1}$.

parameters, the cone half-opening angle $\left(\theta_{\max }\right)$ and the cloud velocities, which are radial and are assumed to be constant with radius $\left(V_{\text {out flow }}\right)$. These are the only two free parameters since the relative geometric orientation of the wind with respect to the quasar line-of-sight is provided by the deep CFHT galaxy image. We allow $\theta_{\max }$ to range from $30-45$ degrees, which is consistent with previous observations (Bouché et al. 2012; Kacprzak et al. 2012). The observed blueshifted $\mathrm{H} \alpha-$ $\mathrm{Mg}$ II relative velocity offset constrains that the modeled outflow cone is pointing towards the observer.

In Figure 3, we show a 48 degree inclined cone with $\theta_{\max }=$ 45 degrees in the plane of the sky. We adopt the convention that the $x$ and $y$ axes represent the plane of the sky and are aligned along the galaxy minor and major axes, respectively, while the quasar sight-line is orthogonal to the sky-plane (zaxis). The grey oval represents the inclined disk and the blue circles represent the conical outflow. The quasar location is shown in red. We show the cloud $z$-velocities as a function of position. We also show the line-of-sight velocity distribution of the clouds at the location of the quasar, which closely mimics the shape and width of the transverse Mg II absorption data. This distribution is convolved with an instrumental resolution of $145 \mathrm{~km} \mathrm{~s}^{-1}$, similar to the LRIS data.

We constrained the model wind velocity by enforcing agreement between the wind model $\mathrm{Mg}$ II absorption profile shape, width, and velocity offset and the observed transverse Mg II absorption profile (see Figure 3). The range of radial model winds speeds that provides the best $\chi^{2}$ fit to the data are $V_{\text {out flow }}=40-80 \mathrm{~km} \mathrm{~s}^{-1}$.

In order to directly compare the bi-conical wind model radial velocities to the measured line-of-sight down-the-barrel velocities, we must deproject the down-the-barrel velocities by the galaxy inclination, which provides the perpendicular velocity component emanating from the galaxy disk (which is equivalent to the radial velocity of the axis of symmetry of the wind model). The range of deprojected down-the-barrel velocities is $V_{d t b}=45-255 \mathrm{~km} \mathrm{~s}^{-1}$, which is consistent with, though possibly faster than, the $V_{\text {out flow }}=40-80 \mathrm{~km} \mathrm{~s}^{-1} \mathrm{de}-$ duced from the bi-conical wind model.

We note that we are unable to reproduce the reddest $\mathrm{Mg}$ II component (also seen in silicon), which could be due to stochastic effects from the number of clouds intercepted along the line-of-sight or to limitations of our constant wind velocity, geometrically symmetric model, and/or the absorption may arise from other sources within the galaxy halo. Our model is not the only possible explanation for the absorption and without a more complex model, it is difficult to conclude the origins of the reddest component, however, our simple model does successfully reproduce the majority of the $\mathrm{Mg}$ II and all of the $\mathrm{Mg}$ I absorption.

\subsection{Galaxy and Halo Gas Metallicities}

We compute the galaxy metallicity using the indicator $N 2=\log [f([\mathrm{NII}] \lambda 6583) / f(\mathrm{H} \alpha)]$, which is equivalent to being the ratio of equivalent widths since both $\mathrm{H} \alpha$ and [NII] are only $20.66 \AA$ apart (at rest wavelengths) and the continuum flux levels are approximately equal. The rest-frame equivalent widths, measured from APO/DIS, of $\mathrm{H} \alpha=58 \pm 2 \AA$ and $[\mathrm{NI}] \lambda 6583=13 \pm 1 \AA$ (shown in Figure 1 ) yield $N 2=-0.65 \pm 0.04$. We apply the $N 2$ metallicity relation $12+\log [\mathrm{O} / \mathrm{H}]=8.90+0.57 \times N 2$ (Pettini \& Pagel 2004), assuming a solar oxygen abundance of $12+\log [\mathrm{O} / \mathrm{H}] \odot=8.736 \pm$ 0.078 (Holweger 2001), and compute a galaxy metallicity of $[\mathrm{O} / \mathrm{H}]=-0.21 \pm 0.08$.

To determine the transverse absorbing-gas properties we Voigt profile fit the HST/COS spectrum of SiII, SiIII, SiIV and Ly $\alpha$ using our software MINFIT (Churchill \& Vogt 2001; Churchill, Vogt, \& Charlton 2001), which incorporates the appropriate COS ISF. In Figure 4, we show the SiII, Si III and Ly $\alpha$ (SiIV is not shown since it is a upper limit) along with the Voigt profile fits. The total column densities are shown in Table 1 The velocity structure of Ly $\alpha$ was established from silicon assuming thermal broadening. We find a $\log N(\mathrm{HI})=18.89 \pm 0.15$.

The Mg II column density was determined by fitting Gaussians to the $\mathrm{Mg}$ II profiles using the velocity structure of the Si II lines from their Voigt profile fits. We applied the curveof-growth to each Mg II Gaussian component using the equivalent widths from the Gaussians and the SiII Doppler $b$ parameters. For the $\mathrm{Mg}$ I, only a single Gaussian fit was statistically required. Given small $W_{r}(\mathrm{Mg} \mathrm{I})$, placing it on the linear part of the curve-of-growth, the column density is independent of $b$. The total column densities are presented in Table 1

We use version 08.00 of Cloudy (Ferland et al. 2013), and a solar abundance pattern, to model the metallicity and ionization conditions of the gas. We apply the standard assumption that the gas is represented by a photoionized uniform slab in ionization equilibrium illuminated with a Haardt \& Madau (2012) ionizing spectrum. The ionization parameters, $U$, and the metallicity of the gas are varied to match the observations 

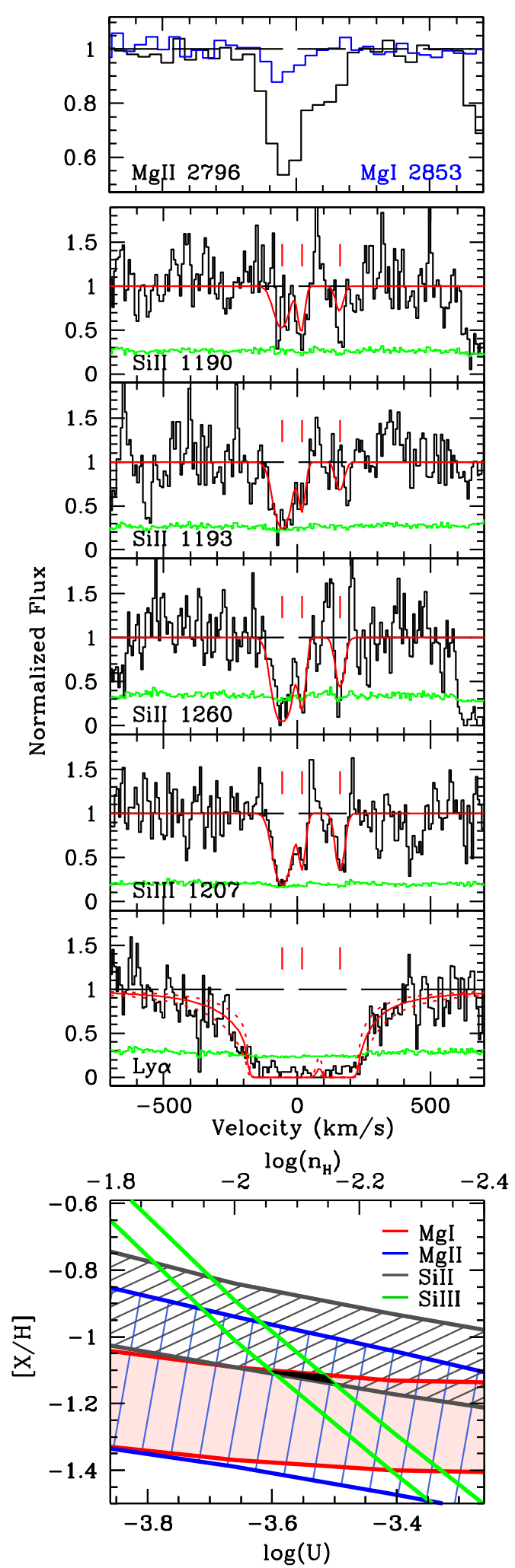

FIG. 4.- (top) Transverse $\operatorname{Mg}$ II $\lambda 2796$ (black) and $\operatorname{Mg} \lambda 22853$ (blue) absorption. (middle) The HST/COS spectrum of the transverse SiII, SiIII and Ly $\alpha$ absorption. The data are shown as a histogram (black) and the sigma spectrum shown below (green). The data have been Voigt profile fit (red), with the number of clouds indicated by the vertical tick-marks. The $\mathrm{N}(\mathrm{HI})=18.89 \pm 0.15$ with the errors on the fit shown as dotted lines. (bottom) The Cloudy models are shown as a function of ionization parameter and metallicity. The upper and lower bounds for each ion include the $\mathrm{N}(\mathrm{X})$ and the $\mathrm{N}(\mathrm{HI})$ errors. The overlapping/common region, indicated by the black shaded region, is where the data constrain the acceptable range of metallicities to be $-1.14 \leq[\mathrm{X} / \mathrm{H}] \leq-1.10$ and ionization parameter to be $-3.60 \leq U \leq-3.50$. of $N(\mathrm{X})$ shown in Table 1. In Figure 4, we show the Cloudy models as a function of ionization parameter and metallicity.

The upper range of $\mathrm{MgI}$ and lower range of SiII enforce $\log U>-3.60$. The steep dependence of SiIII, for which the Voigt profile fit is very robust, further constrains $\log U<-3.50$. Thus, the allowed range of ionization parameter is $-3.60 \leq \log U \leq-3.50$, the range of hydrogen density is $-2.16 \leq n_{H} \leq-2.06$, the metallicity] is $-1.14 \leq$ $[\mathrm{X} / \mathrm{H}] \leq-1.10$, the hydrogen ionization fraction is $-0.77 \leq$ $f(\mathrm{HI}) \leq-0.69$, and the total hydrogen column density is $19.57 \leq \log [\mathrm{N}(\mathrm{H})] \leq 19.68$.

\section{DISCUSSION}

The kinematic models of both the down-the-barrel and transverse absorption produce consistent predictions for the outflow velocities that overlap in the range of $45-80 \mathrm{~km} \mathrm{~s}^{-1}$. These results are highly suggestive that the transverse absorption in the quasar spectrum is physically related to the downthe-barrel absorption via an outflow.

We have computed the galaxy metallicity to be near solar while the transverse absorption at $58 \mathrm{kpc}$ is a tenth solar. The metallicity of the transverse component is consistent with the $0.1-1 Z_{\odot}$ metallicity systems around $\sim 0.1 L_{*}$ galaxies that have velocities consistent with being bound, galactic fountain clouds (Stocke et al. 2013). The absorption-line metallicity is also consistent with the higher metallicity outflowing gas from the bi-modal distribution of Lyman-limit system metallicities from Lehner et al. (2013).

To determine the gas outflow rate we apply Equation 4 of Bouché et al. (2012), derived for a single outflow cone, using our derived model outflow rates and derived $\mathrm{N}(\mathrm{H})$. We estimate the total outflow rate from a bi-conical flow being roughly $1.6-4.2 \mathrm{M}_{\odot} \mathrm{yr}^{-1}$ assuming outflow velocities of $40-80 \mathrm{~km} \mathrm{~s}^{-1}$. With the galaxy having a SFR between 4.6-15 $\mathrm{M}_{\odot} \mathrm{yr}^{-1}$, we conclude that the wind mass-loading factor likely lies in the range of $\eta \sim 0.1-0.9$, which is consistent with loading factors derived for star-forming galaxies at low redshift (Martin 1999; Rupke et al. 2005; Bouché et al. 2012) but slightly lower than recent estimates from scattered $\mathrm{Mg}$ II emission at intermediate redshifts (Martin et al. 2013).

\section{CONCLUSIONS}

We have shown the first example of a galaxy that exhibits absorption observed down-the-barrel and transversely at a projected distance of $58 \mathrm{kpc}$. Both the $\mathrm{Mg}$ II observed downthe-barrel and transversely are blueshifted with respect to the galaxy systemic velocity. We also detect Ly $\alpha$, Si II, Si III, and $\mathrm{Mg}$ I absorption at the transverse location. The quasar sightline resides within $3.5^{\circ}$ of the projected galaxy minor axis where studies suggest that the absorption should be produced by winds.

1. We find that the down-the-barrel deprojected outflow velocities, i.e., perpendicular to the galaxy disk, range between $V_{d t b}=45-255 \mathrm{~km} \mathrm{~s}^{-1}$, which is typical of starforming galaxies (e.g., Martin et al. 2012; Rubin et al. 2013).

2. If we assume a conical outflow model (Bouché et al. 2012), then the constant wind velocities required to reproduce the transverse $\mathrm{MgII}$ absorption kinematics

7 Additional errors from model-dependant assumptions could range between 0.1-0.4dex (Werk et al. 2014). 
are $V_{\text {out flow }}=40-80 \mathrm{~km} \mathrm{~s}^{-1}$, which is consistent with the deprojected down-the-barrel outflow velocities. Although this is a simplistic wind model, our analysis suggests that the absorption is kinematically coupled.

3. We compute the galaxy metallicity to be $[\mathrm{O} / \mathrm{H}]=-0.21 \pm 0.08$, whereas the transverse absorption at $58 \mathrm{kpc}$ has $[\mathrm{X} / \mathrm{H}]=-1.12 \pm 0.02$.

4. The galaxy SFR ranges from 4.6 to $15 \mathrm{M}_{\odot} \mathrm{yr}^{-1}$ while the estimated outflow rate is roughly $1.6-4.2 \mathrm{M}_{\odot} \mathrm{yr}^{-1}$ and yields a wind loading factor of $\eta=0.1-0.9$.

For the first time, we have successfully combined independent models and analysis techniques of down-the-barrel and transverse absorption systems to show that the intrinsic galaxy outflows sufficient to reproduce the observed kinematics of the transverse absorption $58 \mathrm{kpc}$ away. If the metallicty at the base of the outflow equals that of the galaxy ISM, and the wind is continuous, then the observed $\sim 1$ dex decrease in metallicity at $58 \mathrm{kpc}$ suggests that the gas was diluted/mixed with lower metallicty gas. Finding additional systems like this one will aide in our understanding of how outflows transport and redistribute gas within their halos.
We would like to thank the referee for his/her thorough read of the manuscript. We thank Sowgat Mazuhid for providing the HST/COS spectrum. This work was partly supported by a Marie Curie International Outgoing Fellowship (PIOF-GA2009-236012), by a Marie Curie International Career Integration Grant (PCIG11-GA-2012-321702) and by NSF grant AST-1109288. Data was obtained at the W.M. Keck Observatory, which is operated as a scientific partnership among the California Institute of Technology, the University of California and the National Aeronautics and Space Administration. The Observatory was made possible by the generous financial support of the W.M. Keck Foundation. Additional observations were obtained with the APO 3.5-m telescope, which is owned and operated by the Astrophysical Research Consortium. Observations were also made with the NASA/ESA HST or obtained from the data archive at the Space Telescope Institute. This research used the facilities of the Canadian Astronomy Data Centre operated by the National Research Council of Canada with the support of the Canadian Space Agency.

Facilities: Keck I (LRIS), Sloan (SDSS), HST (COS), APO (DIS).

\section{REFERENCES}

Barton, E. J., \& Cooke, J. 2009, AJ, 138, 1817

Bordoloi, R., Lilly, S. J., Hardmeier, E., et al. 2013, arXiv:1307.6553

Bordoloi, R., Lilly, S. J., Kacprzak, G. G., \& Churchill, C. W. 2014, ApJ, 784, 108

Bordoloi, R., Lilly, S. J., Knobel, C., et al. 2011, ApJ, 743, 10

Bouché, N., Hohensee, W., Vargas, R., Kacprzak, G. G., et al. 2012, MNRAS, 426, 801

Bouché, N., Murphy, M. T., Kacprzak, G. G., et al. 2013, Science, 341, 50 Churchill, C. W., \& Vogt, S. S. 2001, AJ, 122, 679

Churchill, C. W., Vogt, S. S., \& Charlton, J. C. 2003, AJ, 125, 98

Coil, A. L., Weiner, B. J., Holz, D. E., et al. 2011, ApJ, 743, 46

Ferland, G. J., Porter, R. L., van Hoof, P. A. M., et al. 2013, Revista Mexicana de Astronomia y Astrofisica, 49, 137

Gwyn, S. D. J. 2012, AJ, 143, 38

Haardt, F., \& Madau, P. 2012, ApJ, 746, 125

Holweger, H. 2001, Joint SOHO/ACE workshop "Solar and Galactic Composition", 598, 23

Kacprzak, G. G., Churchill, C. W., \& Nielsen, N. M. 2012, ApJ, 760, L7

Kacprzak, G. G., Churchill, C. W., Steidel, C. C., Spitler, L. R., \& Holtzman, J. A. 2012, MNRAS, 427, 3029

Kennicutt, R. C., Jr. 1998, ARA\&A, 36, 189

Kornei, K. A., Shapley, A. E., Martin, C. L., et al. 2012, ApJ, 758, 135

Lehner, N., Howk, J. C., Tripp, T. M., et al. 2013, ApJ, 770, 138

Martin, C. L. 1999, ApJ, 513, 156
Martin, C. L. 2005, ApJ, 621, 227

Martin, C. L., Shapley, A. E., Coil, A. L., et al. 2013, ApJ, 770, 41

Martin, C. L., \& Bouché, N. 2009, ApJ, 703, 1394

Martin, C. L., Shapley, A. E., Coil, A. L., et al. 2012, ApJ, 760, 127

Noll, S., Burgarella, D., Giovannoli, E., et al. 2009, A\&A, 507, 1793

Oke, J. B., et al. 1995, PASP, 107, 375

Oppenheimer, B. D., Davé, R., Kereš, D., et al. 2010, MNRAS, 406, 2325

Péroux, C., Bouché, N., Kulkarni, V. P., York, D. G., \& Vladilo, G. 2011, MNRAS, 410, 2237

Pettini, M., \& Pagel, B. E. J. 2004, MNRAS, 348, L59

Press, W. H., Teukolsky, S. A., Vetterling, W. T., \& Flannery, B. P. 1992,

Numerical Recipes, Press Syndicate of the Cambridge University (New York, NY)

Rubin, K. H. R., Prochaska, J. X., Koo, D. C., et al. 2013, arXiv:1307.1476

Rubin, K. H. R., Prochaska, J. X., Koo, D. C., \& Phillips, A. C. 2012, ApJ, 747, L26

Rupke, D. S., Veilleux, S., \& Sanders, D. B. 2005, ApJS, 160, 115

Shen, S., Madau, P., Aguirre, A., et al. 2012, ApJ, 760, 50

Stocke, J. T., Keeney, B. A., Danforth, C. W., et al. 2013, ApJ, 763, 148

Tremonti, C. A., Moustakas, J., \& Diamond-Stanic, A. M. 2007, ApJl, 663, L77

Weiner, B. J., et al. 2009, ApJ, 692, 187

Werk, J. K., Prochaska, J. X., Tumlinson, J., et al. 2014, arXiv:1403.0947 